- RAHIS, Revista de Administração Hospitalar e Inovação em Saúde Vol, I8, n 2 " Belo Horizonte, MG " ABR/JUN 202I "e-ISSN: 2177-

\title{
GASTOS DO SISTEMA PÚBLICO DE SAÚDE COM TRATAMENTO EM ONCOLOGIA
}

PUBLIC HEALTH SYSTEM EXPENDITURES WITH ONCOLOGY TREATMENT

GASTOS DEL SISTEMA PÚBLICO DE SALUD CON TRATAMIENTO ONCOLÓGICO

Helen Maria da Silva Gomes helensgomes@,hotmail.com

João Carlos Hipólito Bernardes do Nascimento Universidade Federal do Piauí joaohipolito@ufpi.edu.br

Anderson Rafael Costa Sousa rafaelcosta.cic@gmail.com

Alysson Nunes de Moura Almeida alynunes@,hotmail.com 


\section{RESUMO}

Objetivo: Identificar e estruturar as despesas do sistema público de saúde com tratamento em oncologia e discutir a respeito dos resultados encontrados em relação aos gastos praticados em outros países. Método: Trata-se de uma pesquisa longitudinal retrospectiva, entre 2008 e 2020. Realizou-se uma análise dos custos de produção ambulatorial do SUS (DATASUS - SAI). Classificou-se os gastos conforme os tipos de tratamento; estas variáveis geraram estatísticas descritivas. Calculou-se, também, uma estimativa do valor médio por sessão de tratamento. Resultados: Verificou-se um aumento crescente, de 2008 a 2020 nos procedimentos clínicos pelo SUS, entre os quais, os gastos com oncologia representavam a segunda maior alocação. Dos gastos totais, os procedimentos de Quimioterapia paliativa - adulto detém maior volume, por outro lado, os menores desembolsos são alocados nos procedimentos de medicina nuclear - terapêutica oncológica. Conclusão: Em relação a países desenvolvidos, o Brasil ainda se apresenta tímido quanto a destinação de recursos à saúde, sobretudo aos procedimentos com tratamento em oncologia.

Palavras-chave: Sistema Público de Saúde. Oncologia. Gastos em Saúde.

\section{ABSTRACT}

Objective: To identify and structure the expenses of the public health system with oncology treatment and discuss the results found in relation to the expenses practiced in other countries. Method: This is a retrospective longitudinal research, between 2008 and 2020. An analysis of the SUS outpatient production costs (DATASUS - SAI) was carried out. Expenditures were classified according to the types of treatment; these variables generated descriptive statistics. An estimate of the average value per treatment session was also calculated. Results: There was an increasing increase from 2008 to 2020 in clinical procedures by SUS, among which, oncology spending represented the second largest allocation. Of total expenditures, palliative chemotherapy procedures - adult holds the largest volume, on the other hand, the lowest disbursements are allocated to nuclear medicine procedures oncology therapy. Conclusion: In relation to developed countries, Brazil is still shy regarding the allocation of resources to health, especially to procedures with treatment in oncology.

Key words: Public Health System. Oncology. Health Expenditure.

\section{RESUMEN}

Objetivo: Identificar y estructurar los gastos del sistema público de salud con tratamiento oncológico y discutir los resultados encontrados en relación con el gasto en otros países. Método: Se trata de una investigación longitudinal retrospectiva, entre 2008 y 2020. Se ha realizado un análisis de los costes de producción de los pacientes externos del SUS (DATASUS - SAI). Los gastos se clasificaron según los tipos de tratamiento; estas variables generaron estadísticas descriptivas. También se calculó una estimación del valor medio por sesión de tratamiento. Resultados: Hubo un aumento creciente de 2008 a 2020 en los procedimientos clínicos por el SUS, entre los cuales, el gasto en oncología representó la segunda mayor asignación. Entre los gastos totales, los procedimientos de quimioterapia paliativa para adultos son los de mayor volumen; por otra parte, los menores desembolsos se destinan a los procedimientos de medicina nuclear - terapéutica oncológica. Conclusión: En relación con los países desarrollados, Brasil aún se muestra tímido en cuanto al destino de los recursos a la salud, sobre todo a los procedimientos con tratamiento en oncología.

Palabras clave: Sistema de salud pública. Oncología. Gastos sanitarios. 


\section{INTRODUÇÃO}

De acordo com Fidler et al. (2017), o câncer é uma das principais causas de morbidade e mortalidade em todas as faixas etárias nas economias desenvolvidas e em transição. Estima-se que a incidência para 2025 seja de 20 milhões de novos casos para o mundo, destes, 16 milhões (80\%) ocorrerão em países em desenvolvimento (AVELLAR et al., 2019). O Instituto Nacional de Câncer (INCA) afirma que os cânceres de brônquios e pulmões, próstata e estômago são os que mais matam homens; enquanto mama, brônquios e pulmões e colo do útero, mulheres (2017).

Consolidou-se no país um sistema híbrido de assistência à saúde, o primeiro público, universal e de custo adicional, além de um sistema privado, compreendendo numerosos provedores e instalações de planos de seguro; ambos operam em paralelo, com serviços organizados de maneira descentralizada. A base brasileira, o SUS, carrega consigo a responsabilidade de fomentar a equidade de acesso à saúde, um direito fundamental expresso na Declaração Universal dos Direitos Humanos (ONU, I948) e social, garantido aos cidadãos brasileiros como dever do Estado, disposto na Constituição Federal de I988 (PAIM et al., 20I I).

A partir do dispositivo constitucional, a Lei n ${ }^{\circ} 8.080 /$ I990 e a Lei Complementar no I4I/20I2 definem a aplicação de recursos mínimos em ações e serviços públicos de saúde a serem investidos pela União, Estados e Municípios; à luz das diretrizes de descentralização, atendimento integral e participação da comunidade. A Lei de Responsabilidade Fiscal e Emenda Constitucional n ${ }^{\circ}$ 29/2000 determinam os mínimos percentuais em relação ao Orçamento que devem ser gastos em saúde; considerando as premissas do planejamento, transparência, controle e responsabilidade que regem a máquina pública administrativa no Brasil (SILVA; VACOVSKI, 2018).

Ressalta-se, portanto, que todas as despesas públicas com saúde, por essência, buscam garantir a democratização do acesso e o bem-estar social frente os óbices de desempenho otimizado provocados pela expansão territorial e distintas realidades socioeconômicas da população. Consoante, o relatório do Banco Mundial dos aspectos fiscais da saúde pública no Brasil aponta desafios estruturais para estimação da qualidade do gasto, uma vez que os custos com serviços de saúde crescem acima da média da inflação, junto ao crescimento da proporção de pessoas idosas, que eleva o dispêndio em valores reais e volumes anuais, em pelo menos 50\% de 2017 se comparado a 2008, considerando que pacientes acima de 50 anos ocupam apenas $22 \%$ da população (BANCO MUNDIAL, 2018).

O controle dos gastos tem se tornado cada vez mais central nos esforços para melhorar a qualidade da saúde. Buscando conhecer, assegurar e melhorar a qualidade do atendimento, governos e outras autoridades têm desenvolvido e ampliado processos e dispositivos contábeis em cada vez mais aspectos da organização médica (PFLUEGER, 20I5). Os gastos com serviços oncológicos no SUS saíram de 470 milhões em 1999 e chegaram a I,6 bilhões em 2007, considerando os serviços divididos em 3 fases: (I) o período posterior ao diagnóstico; (2) a etapa contínua ou fase de monitoramento, que é o tempo entre a fase inicial e a fase final de vida; e (3) o último ano de vida (ELIAS et al., 2020). Categorizando as despesas por procedimentos em oncologia, que vão desde gastos com ações de prevenção até gastos de atenção complementar, identifica-se o desembolso de I,8 bilhões em 2008 e 3,9 bilhões em 2016, aumento gradativo de $120 \%$. Em média, considerando o recorte temporal, gastou-se $59,90 \%$ com procedimentos clínicos e 31,54\% com procedimentos cirúrgicos, necessários à longevidade do ciclo de vida dos pacientes. As despesas menos expressivas estão alocadas nas políticas de prevenção e na compra de medicamentos, com média de $0,40 \%$ e 0,34\%, respectivamente (OBSERVATÓRIO DE ONCOLOGIA, 2018).

Com base no exposto, este artigo tem como objetivo identificar e estruturar as despesas do sistema público de saúde com tratamento em oncologia e discutir a respeito dos resultados encontrados em relação aos gastos praticados em outros países. Este documento visa contribuir para este debate sobre o papel do controle dos gastos na melhoria da qualidade.

Há uma escassez de literatura examinando os gastos contra o câncer no Brasil e explorando seu valor em termos de melhoria da saúde. Em vez disso, a literatura até o momento tem se concentrado no 
valor da perspectiva do tratamento do câncer, agregando o tratamento médico à terapêutica medicamentosa. Usando abordagens convencionais para avaliar vidas estatísticas, alguns relataram que os cuidados oncológicos de alto custo nos Estados Unidos fornecem valor positivo líquido para a sociedade e que gastos mais altos estão correlacionados com ganhos na expectativa de vida.

\section{REFERENCIAL TEÓRICO}

O Sistema Único de Saúde (SUS) surgiu em 1988 unindo serviços da União, Estados, Municípios e da assistência médica previdenciária passou a se tornar um serviço universal, descentralizado e que integra as redes assistenciais como diretrizes fundamentais (SILVA; SILVA; BOUSSO, 20I I). Complementado pela Norma Operacional da Assistência à Saúde (NOAS/200I), que teve como objetivo integrar a saúde de regiões que agregam vários municípios. Em 2019, o serviço apresentou mais de 200 milhões de pessoas beneficiárias (MARQUES et al., 2019).

O SUS é considerado a maior política de inclusão social. Rompeu a divisão ao acesso a uma assistência curativa razoável e transformou a saúde em um direito de todos e dever do Estado. Em 20I3, ao completar 25 anos, apresentava aproximadamente seis mil hospitais e mais de 60 mil ambulatórios contratados, mais de II milhões de internações hospitalares, mais de dois bilhões de procedimentos ambulatoriais, quase IO milhões de procedimentos de quimioterapia e radioterapia, mais de $200 \mathrm{mil}$ cirurgias cardíacas e mais de I50 mil vacinas por ano tornando a prática de programas de referência internacional (MENDES; BITTAR, 20I4).

Apesar do crescimento nas últimas duas décadas, o SUS ainda enfrenta dificuldades, como: o financiamento, o atendimento às necessidades da saúde por toda a população e a escassez de médicos em cidades menos desenvolvidas (SOUZA, 2019). Devido a um plano de organização microeconômica, o SUS não é capaz de responder, com efetividade, eficiência e qualidade, a situação de saúde vigente (MENDES; BITTAR, 2013), em consequência disto, é possível observar, recentemente, em alguns estados, uma alteração na forma de organização do SUS, como a integração em Rede da Atenção Primária (APS) com a Atenção Ambulatorial Especializada (AAE) (DALCUCHE; MENDES, 20I7).

Uma forma recentemente aplicada para desenvolvimento da eficiência, economicidade e facilidade no pronto atendimento é organizar o Sistema de Saúde de forma que serviços gerais, como o de atenção primária à saúde, estejam presentes de forma dispersa, enquanto serviços de maior complexidade estejam concentrados, assim, idealiza-se que o cidadão não busque um serviço de maior aparato tecnológico para a solução de um problema que pode ser resolvido por um serviço de menor porte (SANTOS, 20I7). Um determinante significativo para a cura do paciente em tratamento oncológico é a combinação da radioterapia, cirurgia e tratamento sistêmico perioperatório (KALIKS et al., 20I7), além de um diagnóstico precoce (BRITO; PORTELA; VASCONCELLOS, 2005).

Em I990, o SUS estabeleceu o Instituto Nacional do Câncer (INCA) como responsável por formulação e desenvolvimento de políticas para prevenção e controle da doença (BARRETO, 2005). Em novembro de 1998, através da portaria do Ministério da Saúde n ${ }^{\circ} 3.535$, determinou-se hospitais com estrutura para assistência oncológica no SUS, os Centros de Alta Complexidade em Oncologia (CACON). Em março de 1999, através da Portaria n ${ }^{\circ}$ II3, surgiram Unidades Isoladas (UI) para radioterapia e quimioterapia (BRITO; PORTELA; VASCONCELLOS, 2005).

A partir dos anos 2000, a assistência oncológica fornecida pelo SUS é parte da Autorização de Procedimentos de Alta Complexidade (APAC) (KALIKS et al., 20I7, BARRETO, 2005).

A Política Nacional de Atenção Oncológica foi promovida em dezembro de 2005, reiterando o câncer como um problema de saúde pública (Portaria GM/MS 2.439). Assim, surgiu a Rede de Atenção Oncológica, que estabeleceu um trabalho coletivo entre Governo Federal, Secretarias Estaduais e Municipais de Saúde, universidades, centros de pesquisas, organizações não governamentais e sociedade (BARRETO, 2005). 
Paralelamente, a Secretaria de Assistência à Saúde (SAS) vinculada ao Ministério da Saúde (MS), estabelece diretrizes terapêuticas para diversos tipos de câncer: os Protocolos Clínicos e Diretrizes Terapêuticas (PCDTs), remunerado exclusivamente pelo SUS; e as Diretrizes Diagnósticas e Terapêuticas (DDTs), que levam em conta outras formas de financiamento (KALIKS et al., 20I7).

\section{Intervenção Governamental nas Fases Pós SUS}

A primeira proposta governamental envolvendo oncologia surgiu em 1920, estabelecendo a notificação dos casos e seu registro como causa de óbito (SILVA et al., 2018). A partir da década de 40 começaram a surgir a utilização de propagandas como artigos, filmes e cartazes objetivando informar ao público sobre o câncer. As campanhas eram uma estratégia governamental para identificação, controle e tratamento da enfermidade. Em I94I, ocorreu uma reforma complexa na área da saúde pelo Ministro da Educação e Saúde; dela, surgiram I3 serviços nacionais ligado ao controle de doenças que estavam em alta, entre elas estava o câncer (TEIXEIRA et al., 20I2).

O primeiro regulamento surgiu em novembro de 1993, a Portaria n ${ }^{\circ} 170$ da Secretaria de Assistência à Saúde, que destaca critérios para credenciamento de serviços de alta complexidade em oncologia. A Portaria GM/MS n $3.535 / 98$, estabeleceu os Centros de Alta Complexidade em Oncologia (CACON) e visou fornecer o atendimento integral aos pacientes com câncer (TEIXEIRA et al., 20I2).

Em 1996, a Coordenação Nacional de Controle do Tabagismo e Prevenção Primária do Câncer (CONTAPP), responsável por as primeiras ações como: Dia Mundial do Tabaco (3I de maio) e o Dia Nacional de Combate ao Fumo (29 de agosto). Neste mesmo ano, inicia-se o Programa Controle do Câncer de Colo Uterino - Viva a Mulher o qual objetivava informar e incentivar as mulheres a realizarem o exame de prevenção e detecção da doença (BARRETO, 2005).

O Instituto Nacional do Câncer (INCA) passou a ter competência para o desenvolvimento de campanhas em combate ao câncer a partir de 2000, conforme Decreto Presidencial n ${ }^{\circ} 3.496$, torna-se, assim, referência no SUS para prestação de serviços oncológicos (BARRETO, 2005).

Em dezembro de 2005, a Portaria GM/MS n 2.439 fixou as bases de uma ampla Política Nacional de Atenção Oncológica, esta buscava: (I) promover a qualidade de vida e da saúde da sociedade; (2) organizar linhas de cuidado que envolvam todos os níveis de atenção (básica e especializada, de média e alta complexidades) e de atendimento (promoção, prevenção, diagnóstico, tratamento, reabilitação e cuidados paliativos); e (3) constituir redes hierarquizadas e organizadas, garantindo o acesso e o atendimento integral (TEIXEIRA et al., 20I2).

\section{Desafios do SUS e Despesas com Saúde no Brasil}

Os dados, segundo o Ministério da Saúde, do Relatório de Gestão em Saúde apontaram que o Brasil orçou em 2019 um gasto de 137,8 bilhões de reais para as políticas públicas que permeiam questões sanitárias, epidemiológicas e ambulatoriais (correspondente a mais de $50 \%$ do plano) até demais subfunções de caráter específico do Fundo Nacional de Saúde. Até a data de publicação do documento mais de $10 \%$ da despesa empenhada ainda não havia sido paga, enquanto deteve mais de $15 \%$ de crédito adicional ao longo do exercício. Cumpriu-se no período o teto incluído pela Emenda Constitucional 95, de 15 de dezembro de 2016 - determina aplicação mínima em saúde análoga ao piso apurado em 2018, corrigido pela variação da inflação (IPCA) acumulada em 4,39\% (MINISTÉRIO DA SAÚDE, 2020).

Segundo dados do Instituto Brasileiro de Geografia e Estatística (IBGE, 20I7) as despesas com saúde representam 8,2\% do Produto Interno Bruto (PIB), gasto equiparado ao dos países subdesenvolvidos e expressivos quando não se considera que o sistema de saúde deve garantir cobertura universal e atendimento integral. Para Piola et al (2013), o óbice reside justamente em financiar um sistema assistencialista e íntegro diante de limitações estruturais. Na tentativa de sanar a dificuldade adotaram-se incentivos governamentais ao mercado privado, que, em excesso, ultrapassou o gasto público. 
Assim, o Brasil é atualmente o único país com sistema universal que possui gasto privado superior ao público (PIOLA et al, 2013).

Santos Neto et al (2017) identifica que duas matérias que dificultam não só o gerenciamento dos recursos públicos em saúde, bem como sua manutenção e continuidade, possuem aparato fiscal: (I) fragmentação do sistema universal de saúde diante da autonomia municipal e financiamento heterogêneo, dependente e; (2) renúncia fiscal dada no imposto de renda. Mais de $60 \%$ do orçamento com saúde destina-se a transferências constitucionais aos Estados e Municípios, isso porque a despesa com saúde nessas esferas é composta pela transferência recebida da União e percentual aplicado à receita de impostos; a deficiência na arrecadação acarreta no menor financiamento da saúde com recursos próprios e maior dependência aos recursos da União. Basicamente, o sistema é custeado pelas receitas de impostos gerais, contribuições sociais, desembolsos diretos e demais receitas fiscais (FIGUEIREDO et al; 2018)

Enquanto alguns Estados e Municípios conseguem subsidiar o sistema de saúde, uma parcela significativa ainda não detém desta autonomia, fazendo com que o SUS funcione sempre à margem do desempenho esperado e sob a pressão de corresponder as necessidades sociais. Essa desigualdade na contribuição do custeio do SUS implica gastos ineficientes e não correspondem ao crescimento da Receita Corrente de 6,7\% na última década em relação ao gasto que fora de 3,1\% somente, no mesmo período, segundo relatório de Vieira, Piola e Benevides (2019).

Outro fato prejudicial ao financiamento do sistema de saúde consiste na renúncia de receita de impostos atribuída a dedução, sem limite, de gastos com assistência à saúde no imposto de renda de pessoas físicas e jurídicas. Tal benefício não só cofinancia o sistema privado de saúde como denota, em 2015, por exemplo, gasto tributário de R \$ 16,2 bilhões. Com isso, uma parte minoritária da população, ou pelo menos os que detém maiores rendimentos, conta com subsídio público de cobertura duplicada; cenário inverso ao proposto no SUS, de universalização (OCKÉ-REIS; FERNANDES, 2018).

Dada a vantagem no setor privado chega-se a formatação do gasto per capita do SUS, para toda a população de $\mathrm{R}$ \$ 1.283, enquanto a despesa per capita de planos de saúde e seguros privados fora de R\$3.385 no ano de 2018. Instigada pelas problemáticas levantadas, a literatura desenvolve observações acerca de delimitar a eficiência dos gastos públicos em saúde de forma regionalizada. Andrett et al (2018) concluiu que os desempenhos estão aquém do nível esperado de eficiência embora os gastos permanecem crescentes.

\section{METODOLOGIA}

Trata-se de um estudo longitudinal retrospectivo, pois foram coletados dados de um intervalo passado que compreendeu o período de 2008 a 2020, de natureza descritiva exploratório. Na assistência à saúde, a pesquisa descritiva visa explorar fenômenos encontrados.

A pesquisa tem o objetivo identificar e estruturar as despesas do sistema público de saúde com tratamento em oncologia e discutir a respeito dos resultados encontrados em relação aos gastos praticados em outros países, considerando os custos hospitalares ambulatoriais, por se tratar de categorias e fontes primárias mais relevantes.

Para contabilizar os custos hospitalares, realizou-se uma análise retrospectiva dos custos de produção ambulatorial do SUS (DATASUS - SAI), considerando os tipos de tratamentos pelo SUS, o valor autorizado e apresentado por procedimento. Não foram considerados os dados do Sistema de Informações Hospitalares sobre despesas com Internações devido à desatualização dos dados, os quais os últimos dados divulgados por tipo de despesa correspondiam a dezembro de 2006.

Classificou-se os gastos conforme os tipos de tratamento: radioterapia, quimioterapia e medicina nuclear. Esta classificação permitiu alocar o consumo médio de recursos de cada tipo de tratamento. Estes dados foram digitados em planilhas da Microsoft Excel e, em seguida, transportados para o software $I B M \circledast$ SPSS $®$, versão 25.0, onde geraram estatísticas descritivas, como médias, desvio padrão, mínimos 
e máximos para as variáveis quantitativas; calculou-se, também, uma estimativa do valor médio por sessão de tratamento.

Os dados foram obtidos em março de 202I. Todos os dados foram dispostos de forma a viabilizar uma melhor visualização dos mesmos pelo leitor.

\section{RESULTADOS E DISCUSSÃO DOS RESULTADOS}

\section{Análise dos Dados e Discussão do Tratamento em Oncologia no Brasil}

Quaisquer que sejam as questões a serem tratadas no financiamento e gestão do sistema, o SUS enfrenta problemas de saúde pública e de prestação de serviços ainda mais graves. Apesar dessas questões relacionadas ao acesso, a carga catastrófica de custos de transplantes, tratamentos de câncer, cirurgia cardíaca, cuidados de longo prazo e hemodiálise recai principalmente sobre o sistema público. Essa é uma das razões pelas quais mesmos aqueles que têm cobertura de saúde privada tendem a recorrer ao serviço público quando precisam de serviços caros e sofisticados. O sistema privado (PHI - assistência médica e odontológica) do Brasil cobre aproximadamente 48 milhões de pessoas. $\mathrm{O}$ governo federal ainda concede isenções fiscais destinadas a incentivar famílias e empregadores a retirarem PHI. Isso representa uma adaptação à inadequação do sistema público (OCKÉ-REIS; MARMOR, 20I0).

Nesse contexto, verifica-se um aumento crescente, de 2008 a 2020, em todos os procedimentos clínicos. Em 2008, o maior valor gasto foi em tratamento em nefrologia, de R \$ 1.351.622.701,90; seguido por tratamento em oncologia, de R \$ 1.233.359.292,78; e por outras especialidades de tratamento clínico, R \$251.859.945,56. Em 2020, manteve esta ordem para maiores gastos, com os seguintes valores: R \$ 3.2I7.I75.676,64; R \$ 2.602.285.739,20; e R \$ 297.859.258,90, respectivamente (Figura 0I).

\section{Figura 0I: Valor gasto de 2008 a 2017 com procedimentos clínicos pelo SUS.}

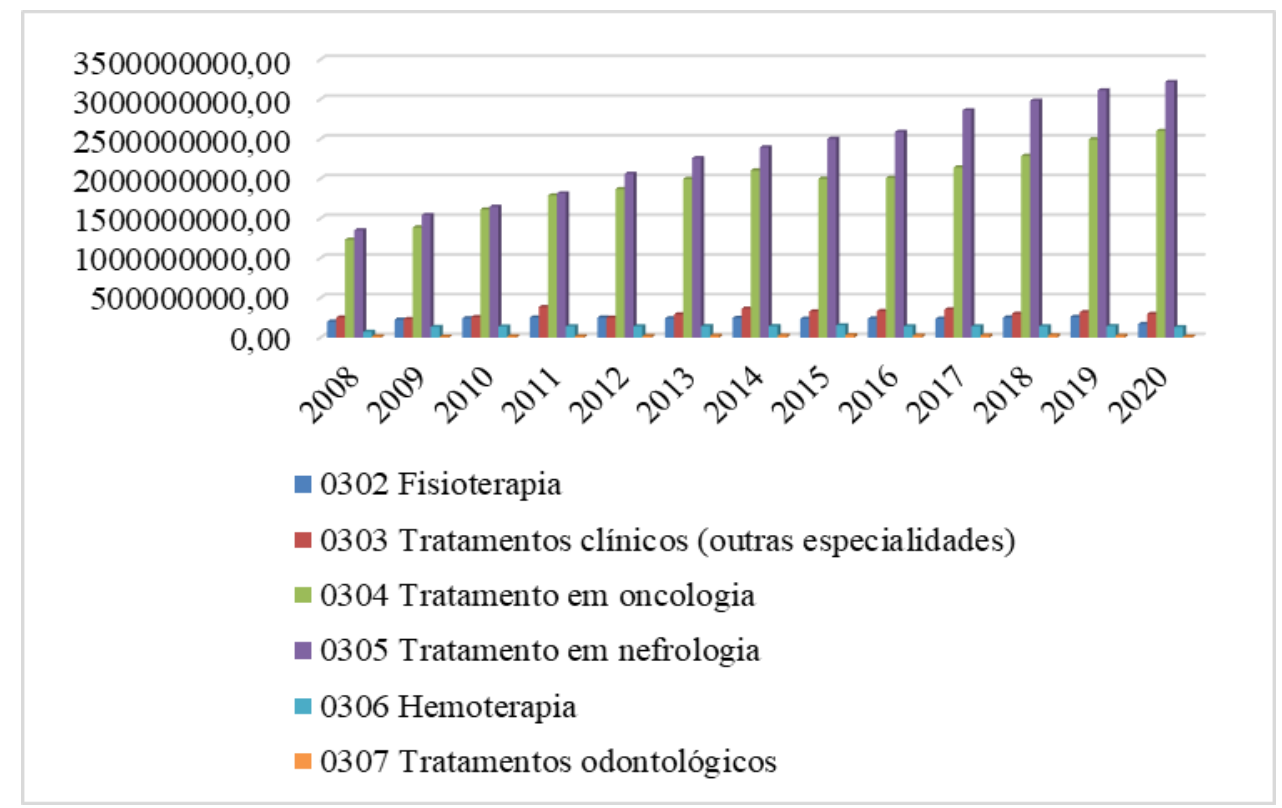

Fonte: Elaborada pelos autores.

Os menores gastos foram constatados, em 2008, em tratamentos odontológicos, R $\$$ I5.983.I69,I7; terapias especializadas, R \$ 39.I7I.8I3,62; e hemoterapia, R \$ 72.276.842,I8. Em 20I7, manteve-se esta ordem e alcançou os valores de: $R \$ 25.619 .737,67$; $R$ \$ 46.400.173,I3; e $R \$$ I42.9I8.I85,23, respectivamente (Figura 03). 
O programa público não atraiu a força de trabalho assalariada ao oferecer uma gama de serviços de saúde abrangentes, em particular, consultores especializados, exames diagnósticos, terapias avançadas (serviços ambulatoriais) e cirurgias eletivas (leitos de internação). Os subsídios fiscais públicos de seguros privados de saúde privam o SUS de recursos financeiros que poderiam ser usados para melhorar seu acesso e qualidade. Enquanto isso, pacientes com seguro privado de risco ruim e doenças crônicas são expulsos do PHI para o SUS, sem qualquer impacto significativo nos lucros das seguradoras de saúde (OCKÉREIS; MARMOR, 20I0).

Independentemente do fato de que gastos excessivos com saúde e gastos catastróficos em saúde tenham sido definidos e medidos de forma inconsistente em uma série de estudos internacionais, há evidências crescentes de que ambos são fatores que podem impactar negativamente o alcance da equidade em saúde e podem contribuir para o empobrecimento de grupos populacionais, especialmente aqueles mais abaixo na escala social (BARROS; BASTOS; DÂMASO, 20I I).

As modalidades tradicionais de tratamento do câncer incluem terapia de radiação, cirurgia, quimioterapia e terapia de prótons (BIDRAM et al., 2019). A radioterapia desempenha um papel fundamental nos planos de tratamento do câncer, atuando como um curativo essencial e como um tratamento paliativo. No entanto, o Brasil enfrenta uma escassez de acesso ao tratamento por radiação, devido à falta de pessoal treinado e unidades de tratamento suficientes (MENDEZ et al., 2018).

Quanto aos procedimentos de tratamento em oncologia, o maior volume de despesa fora encontrado em Quimioterapia paliativa - adulto, seguido por Radioterapia e Quimioterapia adjuvante (profilática) - adulto. Por outro lado, o menor volume apresentado foi com medicina nuclear - terapêutica oncológica, o qual começou a ser contabilizado em 20I4, seguido por Quimioterapia - procedimentos especiais e Quimioterapia curativa - adulto (Tabela OI).

Tabela 0I: Valor apresentado por procedimento de tratamento em oncologia.

\begin{tabular}{|c|c|c|c|c|c|}
\hline Procedimento & Mínimo & Máximo & Média & Desvio padrão & $\begin{array}{c}\text { Média por } \\
\text { quantidade de } \\
\text { procedimentos }\end{array}$ \\
\hline Radioterapia & $\begin{array}{c}\mathrm{R} \$ \\
\mathrm{I} 53.244 .495,99\end{array}$ & $\begin{array}{c}\mathrm{R} \$ \\
594.539 .868,00\end{array}$ & $\begin{array}{c}\mathrm{R} \$ \\
378.131 .439,29\end{array}$ & $\begin{array}{c}\mathrm{R} \$ \\
\mathrm{I} 32.836 . \mathrm{I} 29,62\end{array}$ & $\begin{array}{c}\mathrm{R} \$ \\
43,74\end{array}$ \\
\hline $\begin{array}{l}\text { Quimioterapia } \\
\text { paliativa - adulto }\end{array}$ & $\begin{array}{c}\mathrm{R} \$ \\
420.298 .998,4 \mathrm{I}\end{array}$ & $\begin{array}{c}\mathrm{R} \$ \\
940.193 .726,90\end{array}$ & $\begin{array}{c}\mathrm{R} \$ \\
700.766 .081,44\end{array}$ & $\begin{array}{c}\mathrm{R} \$ \\
\mathrm{I} 59.533 .9 \mathrm{I} 4,34\end{array}$ & $\begin{array}{c}\mathrm{R} \$ \\
659,78\end{array}$ \\
\hline $\begin{array}{l}\text { Quimioterapia para } \\
\text { controle temporário } \\
\text { de doença - adulto }\end{array}$ & $\begin{array}{c}\mathrm{R} \$ \\
98.903 .236,80\end{array}$ & $\begin{array}{c}\mathrm{R} \$ \\
321.920 .161,05\end{array}$ & $\begin{array}{c}\mathrm{R} \$ \\
\text { I7I.637.540,4I }\end{array}$ & $\begin{array}{c}\mathrm{R} \$ \\
77.347 .283,77\end{array}$ & $\begin{array}{c}\mathrm{R} \$ \\
77 \mathrm{I}, 98\end{array}$ \\
\hline $\begin{array}{l}\text { Quimioterapia } \\
\text { prévia } \\
\text { (neoadjuvante/ } \\
\text { citorredutora)- } \\
\text { adulto }\end{array}$ & $\begin{array}{c}\mathrm{R} \$ \\
\mathrm{I} 23.268 .835, \mathrm{I} 5\end{array}$ & $\begin{array}{c}\mathrm{R} \$ \\
254.348 .020,35\end{array}$ & $\begin{array}{c}\mathrm{R} \$ \\
\mathrm{I} 75.077 .4 \mathrm{I} 2,63\end{array}$ & $\begin{array}{c}\mathrm{R} \$ \\
4 \mathrm{I} .576 .055,35\end{array}$ & $\begin{array}{c}\mathrm{R} \$ \\
\mathrm{I} .254,22\end{array}$ \\
\hline $\begin{array}{l}\text { Quimioterapia } \\
\text { adjuvante } \\
\text { (profilática) - } \\
\text { adulto }\end{array}$ & $\begin{array}{c}\mathrm{R} \$ \\
97.355 .933,50\end{array}$ & $\begin{array}{c}\mathrm{R} \$ \\
313.524 .417,26\end{array}$ & $\begin{array}{c}\mathrm{R} \$ \\
227.9 \mathrm{I} 6 . \mathrm{II} 4,69\end{array}$ & $\begin{array}{c}\mathrm{R} \$ \\
72.777 .548,98\end{array}$ & $\begin{array}{c}\mathrm{R} \$ \\
2 \mathrm{I} 3,58\end{array}$ \\
\hline $\begin{array}{l}\text { Quimioterapia } \\
\text { curativa - adulto }\end{array}$ & $\begin{array}{c}\mathrm{R} \$ \\
44.991 .499,62 \\
\end{array}$ & $\begin{array}{c}\mathrm{R} \$ \\
\mathrm{I} 45.047 .423, \mathrm{I} 8 \\
\end{array}$ & $\begin{array}{c}\mathrm{R} \$ \\
\mathrm{I} 00.984 .603,33 \\
\end{array}$ & $\begin{array}{c}\mathrm{R} \$ \\
34.467 .05 \mathrm{I}, 46 \\
\end{array}$ & $\begin{array}{c}\mathrm{R} \$ \\
\mathrm{I} .788,52 \\
\end{array}$ \\
\hline $\begin{array}{l}\text { Quimioterapia de } \\
\text { tumores de criança e } \\
\text { adolescente }\end{array}$ & $\begin{array}{c}\mathrm{R} \$ \\
8 \mathrm{I} . \mathrm{I} 64.7 \mathrm{I} 2,76\end{array}$ & $\begin{array}{c}\mathrm{R} \$ \\
\text { I59.I } 80.875,92\end{array}$ & $\begin{array}{c}\mathrm{R} \$ \\
\text { II } 8.583 .232,50\end{array}$ & $\begin{array}{c}\mathrm{R} \$ \\
25.296 .334,45\end{array}$ & $\begin{array}{c}\mathrm{R} \$ \\
\mathrm{I} .906,32\end{array}$ \\
\hline $\begin{array}{l}\text { Quimioterapia - } \\
\text { procedimentos } \\
\text { especiais }\end{array}$ & $\begin{array}{c}\mathrm{R} \$ \\
58.473 .019,50\end{array}$ & $\begin{array}{c}\mathrm{R} \$ \\
\mathrm{I} 05.462 .745,50\end{array}$ & $\begin{array}{c}\mathrm{R} \$ \\
91.017 .062,73\end{array}$ & $\begin{array}{c}\mathrm{R} \$ \\
\mathrm{I} 4.83 \mathrm{I} .3 \mathrm{I} 8,38\end{array}$ & $\begin{array}{c}\mathrm{R} \$ \\
496,54\end{array}$ \\
\hline $\begin{array}{l}\text { Medicina nuclear - } \\
\text { terapêutica } \\
\text { oncológica }\end{array}$ & $\mathrm{R} \$$ & $\begin{array}{c}\mathrm{R} \$ \\
297.220,50\end{array}$ & $\begin{array}{c}\mathrm{R} \$ \\
\mathrm{I} 12.674,60\end{array}$ & $\begin{array}{c}\mathrm{R} \$ \\
\text { I I } 7.808,09\end{array}$ & $\begin{array}{c}\mathrm{R} \$ \\
53 \mathrm{I}, 87\end{array}$ \\
\hline
\end{tabular}

Fonte: Elaborada pelos autores. 
Segundo Zaremba et al. (2019), o gasto total mensal em média com um paciente em tratamento ou acompanhamento de câncer é de aproximadamente $\mathrm{R}$ \$ 290,4I por mês e R \$ 3I2,65 para seus cuidadores. Para uma população com renda familiar média de dois salários-mínimos, isso representa um alto impacto na organização financeira pessoal. Essa situação torna difícil para o paciente arcar com os custos do bolso do tratamento e, simultaneamente, cumprir com suas outras obrigações financeiras, resultando em estresse. O estudo de Zaremba et al. (2019) relatou que os pacientes mais suscetíveis a dificuldades financeiras eram aqueles muito idosos ou jovens, ativamente empregados no diagnóstico, com baixo suporte social e pouca economia.

Além disso, o estresse financeiro pode afetar negativamente a qualidade de vida dos pacientes e de seus cuidadores. Os custos do bolso do tratamento podem, ainda, levar os pacientes a não aderirem adequadamente ao seu tratamento, com consequências possivelmente prejudiciais para o seu tratamento antitumoral.

A estratégia de tratamento padrão para câncer localmente avançado é a quimiorradiação concomitante. Apesar desse tratamento, muitos pacientes apresentarão recidiva e alguns também apresentam doença metastática no momento do diagnóstico e são tratadas com quimioterapia paliativa. Recentemente, a adição de uma terapia molecular (bevacizumabe) à quimioterapia paliativa em pacientes com câncer recorrente, persistente ou metastático foi associada a uma melhora de 3,7 meses na sobrevida global. No entanto, estudos apontam que adicionar bevacizumabe à quimioterapia não seria custobenefício nos Estados Unidos se o preço e a dose atuais do medicamento forem mantidos (SANTOS et al., 2019). Entretanto, conforme o demonstrado nesse estudo, observou-se que a média total dos valores nesse tipo de tratamento foi de $\mathrm{R} \$ 53 \mathrm{I}, 87$, que, apesar de ser um valor significativo, já que se refere à sessão, é o quarto menor custo por sessão de procedimento. Todavia, vale ressaltar que essa média é apenas uma estimativa, pois esses valores variam conforme as peculiaridades dos pacientes.

Os gastos, nesse estudo, com quimioterapia paliativa adulta representaram a maior parcela dos custos totais (35,68\%), seguido por radioterapia (19,25\%). Hoje, no Brasil, há um déficit de 255 serviços de radioterapia e está em andamento um plano de investimentos para ampliar o acesso à radioterapia. Cerca de US\$ I55 milhões foram investidos na aquisição de novos equipamentos entre 2017 e 2018 , que, se contabilizados, aumentaria ainda mais a parcela do custo desse tratamento (SANTOS et al., 2019).

A partir do recorte temporal, averiguou-se uma relação estável do volume de gastos, uma vez que nos anos de 2008, 2009 e 2010 os percentuais de despesas em relação ao total fora de 20,22\%, 20,13\% e 2I,04\%; ao ponto que nos anos de 2018 a 2020 os percentuais foram de 20,65\%, 2I,03\% e 25,I4\%. Essa variação é mais significativa no ano de 2020, conforme o ilustrado na Figura 02:

Figura 02: Percentual de crescimento ao gasto com procedimentos de oncologia quanto ao gasto total com procedimentos em saúde.

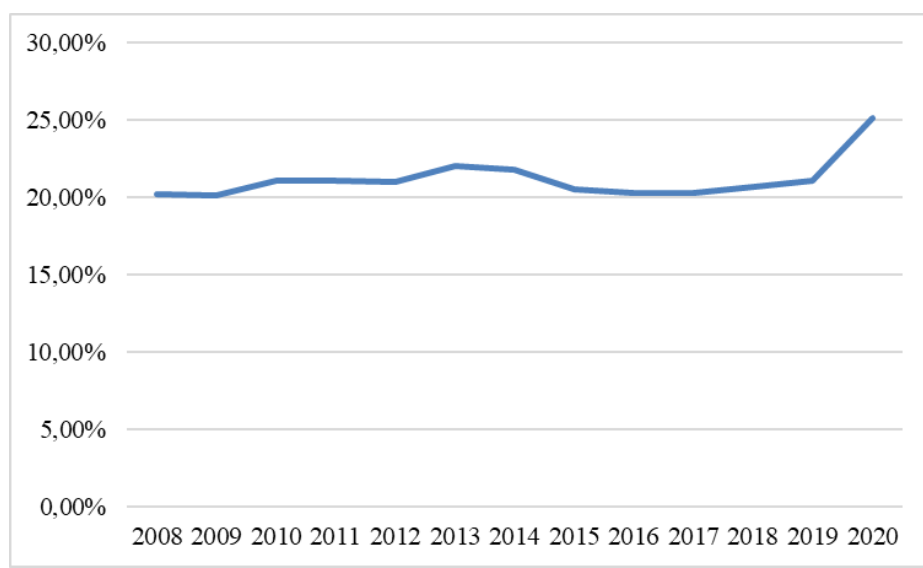

Fonte: Elaborada pelos autores. 
O ônus econômico do tratamento do câncer é alto em todo o mundo, mas não está distribuído igualmente por todas as nações. Apesar do fato de os países de baixa e média renda representarem $84,5 \%$ da população mundial e 61,3\% dos novos casos de câncer em todo o mundo, essas áreas respondem por apenas $6,2 \%$ dos gastos financeiros com câncer (SANTOS et al., 2019). O aumento da expectativa de vida e da população com 60 anos ou mais foi responsável pela maior incidência de câncer nos países em desenvolvimento. Mais de 60\% dos casos de câncer ocorrem na África, Ásia, América Central e América do Sul, e 70\% das mortes por câncer em todo o mundo ocorrem nessas regiões. A Organização Mundial de Saúde (OMS) estimou que esses números podem aumentar ainda mais devido à falta de detecção precoce e acesso insuficiente ao tratamento (SIQUEIRA et al., 20I7).

Dados da OMS mostram US\$ I.085 gastos em saúde per capita em 2013 no Brasil, 48,2\% desses gastos são gastos governamentais. Os EUA (Estados Unidos da América) fora o país com maior gasto em saúde do mundo, com gastos anuais de US\$ 9.I46 per capita no mesmo período, representando I7,I\% do PIB. A estimativa do impacto econômico do câncer no sistema de saúde brasileiro é uma preocupação crescente, principalmente nos dias atuais com o aumento da mortalidade e da incidência de neoplasias no Brasil (SIQUEIRA et al., 20I7).

As mortes prematuras por câncer são uma das prioridades a serem discutidas no Brasil. O custo da mortalidade por anos de vida perdidos (YLL) por câncer é um dos mais altos em comparação com outros países, estimou o custo do câncer nos EUA em 2009 e no estudo de Siqueira et al. (2017) o custo da mortalidade representa 35\% do custo total (CORREA-GALENDI et al., 2020).

Outro fator analisado é que em todos os anos o desembolso efetivo supera o valor orçado. A maior disparidade é dada em 2013, com uma diferença de $\mathrm{R} \$ 29.490 .172,98$ entre o orçado e o apresentado, seguido por 20I4, com $R \$ 27.940 .360,35$ e 20I2, com uma variação de $R \$ 27.938 .525$,86 (Figura 03).

Figura 03: Produção ambulatorial do tratamento em oncologia no SUS de 2008 a 2020.

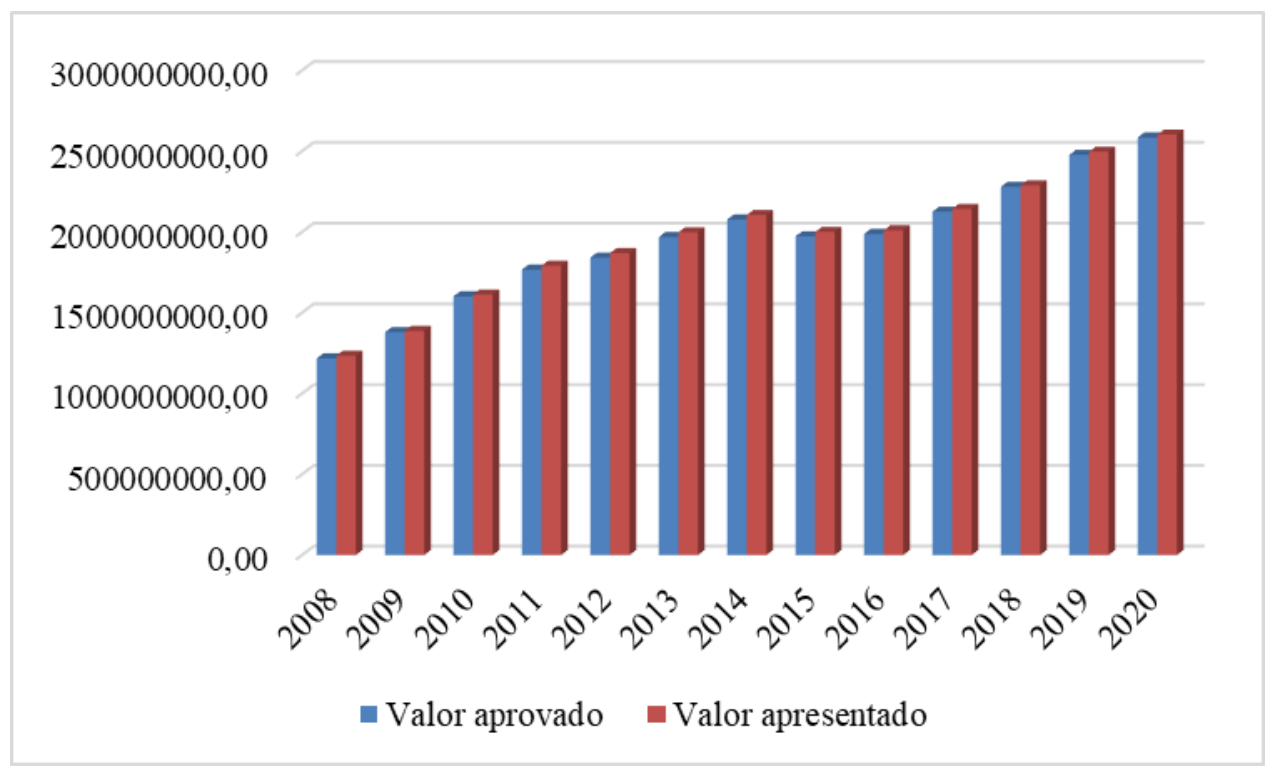

Fonte: Elaborada pelos autores.

A gestão pública brasileira detém de um teto orçamentário em cada estado ou município, quando o custo atinge o limite estabelecido o faturamento é rejeitado. Por esse motivo, a estimativa de custo do câncer neste estudo é considerada conservadora (SIQUEIRA et al., 2017). De acordo com o Banco Mundial, em 2010 os custos com saúde representaram 17,02\% nos EUA, I0,2\% na UE, 9,5I\% no Reino Unido, 8,27\% no Brasil e 6,79\% na Coreia do Sul (CORREA-GALENDI et al., 2020). A razão custo-efetividade calculada no estudo de Correa-Galendi et al. (2020) chegou à conclusão de que está abaixo do limite de custo-efetividade proposto pela Organização Mundial da Saúde (OMS) recomendado 
para países de renda média (ou seja, I a 3 do PIB per capita). A relação entre o teto orçamentário estabelecido e o percentual do PIB gasto com saúde pode refletir, com o passar dos anos, diminuindo, ainda mais a diferença quando comparado a outros países.

Observou-se ainda que o complemento do gestor esteve presente a partir de 2012 e cessou em 20I8. Alcançou um máximo de R \$ 853.450,I5, em 20I3; seguido por 20I4, com R $\$ 776.192,21$ e 20I2, R \$ 7I2.0I5,I3; após esse período, observou-se, na maioria dos procedimentos, uma redução no complemento. Não se identificou complemento federal, conforme figura 04:

Figura 04: Complemento do gestor na produção ambulatorial do tratamento em oncologia no SUS de 2012 a 2018.

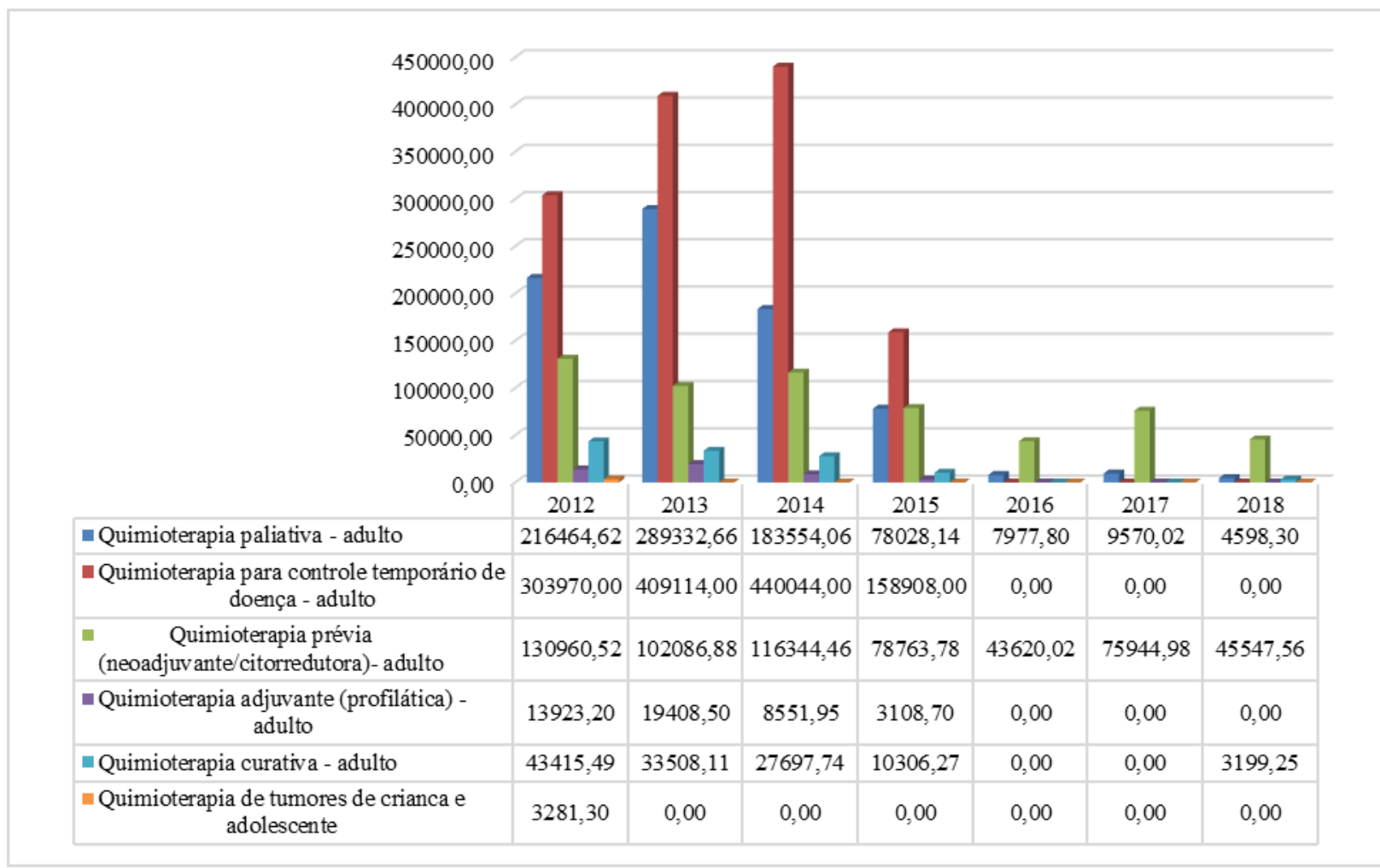

Fonte: Elaborada pelos autores.

O sistema de saúde brasileiro é financiado por meio de impostos, contribuições sociais (impostos para programas sociais específicos), despesas diretas e despesas de saúde dos empregadores, os quais são provenientes da arrecadação de tributos e contribuições sociais dos orçamentos federal, estadual e municipal. Além disso, outras fontes de financiamento são privadas, ou seja, gastos diretos e gastos do empregador. Contudo, como o observado, não têm sido suficientes para garantir recursos financeiros adequados ou estáveis para o sistema público.

As contribuições sociais têm sido maiores do que as contribuições fiscais, que são divididas entre os governos federal, estadual e municipal, o SUS continua subfinanciado. Em 2006, a receita de contribuição social (17,7\%) ultrapassou a receita tributária (I6,7\%). Na esfera federal, as contribuições sociais representam cerca de $60 \%$ da receita tributária geral e menos de $30 \%$ da receita tributária. Além disso, o governo federal recebe $58 \%$ da receita tributária, enquanto os governos estaduais recebem $24,7 \%$ (PAIM et al., 20I I). Em 2007, destinou-se apenas 8,4\% do produto interno bruto para as despesas com saúde; a parcela pública estabeleceu-se acerca de $4 \mathrm{I} \%$, abaixo dos percentuais internacionais encontrados nos países como o Reino Unido (82\%), Itália (77,2\%) e Espanha (7I,8\%), e também inferior ao de países como os EUA (45,5\%) e o México (46,9\%). Fontes privadas de financiamento - gastos diretos de 
famílias e empresas, com subsídios governamentais diretos e indiretos - financiam a maioria dos cuidados de saúde privados planos, apólices de seguro e compra de medicamentos (PAIM et al., 20II).

O gasto direto como proporção do gasto total varia pouco entre as classes mais pobres (5,33\%) e mais ricas $(8,31 \%)$. No entanto, existem diferenças em como cada grupo gasta esses fundos; os mais pobres gastam mais com medicamentos, enquanto os mais ricos gastam mais com planos e seguros privados de saúde (PAIM et al., 20II). Assim, o SUS tem feito menos para aumentar o financiamento público para as necessidades de saúde das pessoas do que o previsto quando foi criado com esse objetivo de estabelecer um sistema de saúde universal e equitativo no Brasil com recursos públicos.

\section{Discussão Internacional}

Enquanto economias desenvolvidas e em transição destinam recursos à saúde na base média de I5 a I8\% do Produto Interno Bruto, o Brasil aloca apenas 8\%. O dado torna-se ainda mais preocupante quando são postos na balança o fato de que o sistema público de saúde brasileiro é dever do Estado por prerrogativa constitucional, ou seja, todo cidadão tem o direito de acesso à saúde recaindo sobre ele a responsabilidade da equidade e universalidade (PIOLA et al, 2013). A dependência de Estados e Municípios da transferência de recursos pela União para garantir as políticas públicas de saúde, uma vez que pela estrutura fiscal e deficiência arrecadatória dos entes vê-se que a autonomia não garante investimentos plenos em boa parte dos municípios, dificultam o financiamento do SUS. Além da renúncia de impostos, dado pela redução, sem limites, das despesas com saúde no imposto de renda que provoca duplo benefício a uma parcela menor da sociedade (SANTOS NETO et al, 2017). O aumento da demanda por tratamento oncológico, juntamente com seu alto custo, impõe uma pressão fiscal significativa sobre o governo, bem como dificuldades financeiras para as famílias. Além disso, a introdução de várias novas tecnologias de tratamento, medicamentos e diagnósticos aumentaram o custo.

No período de 2004 a 20I4, os gastos globais com medicamentos para o tratamento de câncer contribuíram significativamente para a melhoria da sobrevida do paciente. Entretanto, embora os dispêndios continuem a resultar em um valor líquido positivo, os países devem permanecer cautelosos, pois continuam a promover uma agenda de política farmacêutica baseada em valor. Os gastos globais com medicamentos não genéricos utilizados neste tratamento atingiram novos patamares, mesmo com a queda dos volumes. Ainda assim, o aumento no consumo de medicamentos genéricos provoca o desembolso global representativa (SALAS-VEJA; MOSSIALOS, 2016). No Brasil, os novos medicamentos no tratamento do câncer, normalmente, são caros e não estão disponíveis no SUS, pacientes recorrem à Justiça para tentar acesso a essa medicação. Em 2016, foram gastos US \$3.167.701,86 com o processo judicial para recebimento de novos medicamentos contra o câncer (SANTOS et al., 2019).

A radioterapia paliativa, uma das formas apontadas nesse estudo como medida de tratamento pelo SUS, é eficaz no controle rápido de muitos sintomas de cânceres avançados. Seu uso no contexto de pacientes é especialmente relevante, dadas as longas distâncias necessárias para o tratamento especializado do câncer e a maior probabilidade de doença em estágio avançado na apresentação. Embora algumas taxas básicas de utilização de radioterapia entre as populações tenham sido relatadas, não se sabe quanto tempo os pacientes esperam para ter acesso à radioterapia, quanto tempo eles permanecem longe de suas comunidades de origem e o processo de acompanhamento. A literatura cita que 34 a $50 \%$ é tratada por radioterapia paliativa (CHAN et al., 2020).

Estudos sugerem que o gasto durante a fase inicial do atendimento é maior para pacientes em estágio IV do que com doença localizada. Todavia, os esforços anteriores para caracterizar os gastos com câncer avançado limitam-se ao foco predominante em pacientes com doença de metastática (em grande parte devido à falta de dados de status de recorrência na maioria dos registros de tumor). Além disso, as pesquisas já realizadas não explanam os gastos durante todo o episódio de atendimento. Pacientes com câncer recorrente podem incorrer em custos substanciais para tratamento / vigilância do câncer primário localizado (HASSETT et al., 2019). Os gastos com esses serviços, que ocorrem antes do diagnóstico de 
câncer avançado, afetam as estimativas de gastos do tratamento ao longo da vida e dos gastos com doenças avançadas.

\section{CONCLUSÃO}

À medida que as pressões financeiras aumentam, há um debate crescente sobre o valor dos gastos com saúde. O tratamento do câncer é particularmente controverso, tendo em vista que o câncer é a doença mais cara de se tratar e seu tratamento tem sido historicamente um dos principais motores do crescimento dos gastos com saúde. Nesse contexto, o objetivo desse estudo foi identificar e estruturar as despesas do sistema público de saúde com tratamento em oncologia e discutir a respeito dos resultados encontrados em relação aos gastos praticados em outros países.

Os gastos crescentes contra o câncer estão levantando questões sobre o valor para os pacientes e a sociedade. A pressão imposta ao SUS fez com que incentivos fossem liberados à iniciativa privada no setor de saúde, tal fato levou a média per capita de gasto em saúde no setor privado superior ao praticado no setor público. Os relatórios do Ministério da Saúde e achados do referido trabalho apontam que em 2008 destinava-se aproximadamente 600 milhões de reais para as políticas de saúde chegando a 3 bilhões de reais em 2017. Ainda que, segundo pesquisas, o percentual gasto ainda não acompanhou o crescimento da Receita Corrente do Estado (PIOLA; BENEVIDES; VIEIRA, 20I8).

Atrás apenas das despesas com procedimentos clínicos de Nefrologia, pelo SUS, o tratamento em Oncologia cresceu de I5 a I7\% em relação ao gasto total com saúde, o percentual sofre alterações quando são adicionados os gastos com prevenção, consoante levantamento elaborado pelo Observatório de Oncologia (2017). Dos desembolsos com os procedimentos observados no corrente estudo, Quimioterapia paliativa - adulto, seguido por Radioterapia e Quimioterapia adjuvante (profilática) adulto detém maior volume, por outro lado, os menores gastos são alocados nos procedimentos de medicina nuclear - terapêutica oncológica, o qual começou a ser contabilizado em 20I4, seguido por Quimioterapia - procedimentos especiais e Quimioterapia curativa - adulto. Para tal, não se incluiu os custos dos equipamentos de radioterapia, uma vez que foram adquiridos há mais de I0 anos. Hoje, no Brasil, há um déficit de 255 serviços de radioterapia e está em andamento um plano de investimentos para ampliar o acesso à radioterapia.

Em todos os anos observados as despesas apresentadas foram maiores do que a despesa orçada para o período. O complemento do gestor apresentou-se a partir de 2012. Alcançou um máximo de $\mathrm{R} \$$ 440.044,00, em 20I4, com Quimioterapia para controle temporário de doença - adulto; seguido por 2013 e 2012; após esse período, observou-se, na maioria dos procedimentos, uma redução no complemento. Não se identificou complemento federal.

Quando a estrutura de gastos é discutida no âmbito internacional, além dos óbices estruturais apresentados ter-se-á que uma característica difere o Brasil das outras economias do mundo, principalmente os que também adotam um sistema universalizado, que investem de maneira mais agressiva, considerando o percentual sobre o PIB, por exemplo, além da gestão governamental mais eficiente; os investimentos em tecnologia e em pesquisa que contribuem para a longevidade de vida dos pacientes.

O trabalho limitou-se à estruturação e evidenciação dos gastos com procedimentos clínicos em oncologia, o seu comportamento no período investigado e variáveis que possam ter interferido ou comparado aos achados tecendo uma discussão. Os dados foram explorados e estruturados a partir do banco do DataSUS. Portanto, não foram consideradas questões como corrupção política e desvios de recursos da saúde, tampouco a distribuição de recursos por fundos vinculados ao Ministério da Saúde. Outra limitação refere-se à desagregação dos dados, a forma que os dados são dispostos pelo sistema DATASUS não viabiliza a análise relacionando às características da população, como: idade, sexo, escolaridade e etnia.

Considerando o exposto, recomenda-se para estudos futuros a investigação da eficiência dos gastos com tratamento em oncologia através da Análise por Envoltória de Dados (DEA) em função da 
taxa de óbitos por câncer. Bem como a relação/inferência existente entre os gastos com prevenção, tratamento e resultados indesejados, mortalidade. Espera-se que a contribuição incentive a continuidade de pesquisas na temática.

\section{REFERÊNCIAS}

ANDRETT, Marcia et al. Eficiência dos gastos públicos em saúde no Brasil: estudo sobre o desempenho de estados brasileiros. Revista de Gestão em Sistemas de Saúde, v. 7, n. 2, p. I I4-I28, 2018.

AVELLAR, W. O. et al. Cancer research in Brazil: Analysis of funding criteria and possible consequences. Journal of Cancer Policy, v. 20, p. I00184, 2019.

Banco Mundial. Relatório: Aspectos Fiscais da Saúde no Brasil. 2018. Disponível em: $<$ https://www.who.int/health_financing/documents/health-expenditure-report-2018/en/>. Acesso em: 06 de abril de 202I.

BARRETO, Eliana Maria Teixeira. Acontecimentos que fizeram a história da oncologia no Brasil: Instituto Nacional de Câncer (INCA). Revista brasileira de cancerologia, v. 5I, n. 3, p. 267-75, 2005.

BARROS, A. JD; BASTOS, J. L.; DÂMASO, A. H. Catastrophic spending on health care in Brazil: private health insurance does not seem to be the solution. Cadernos de saude publica, v. 27, p. s254s262, $201 \mathrm{I}$.

BIDRAM, E.; ESMAEILI, Y.; RANJI-BURACHALOO, H.; AL-ZAUBAI, N.; ZARRABI, A.; STEWART, A.; DUNSTAN, D. E. A concise review on cancer treatment methods and delivery systems. Journal of Drug Delivery Science and Technology, p. I0I350, 2019.

BRITO, Cláudia; PORTELA, Margareth Crisóstomo; VASCONCELLOS, Mauricio Teixeira Leite de. Assistência oncológica pelo SUS a mulheres com câncer de mama no Estado do Rio de Janeiro. Revista de Saúde Pública, v. 39, p. 874-88I, 2005.

CHAN, J. et al. Time to diagnosis and treatment with palliative radiotherapy among inuit patients with cancer from the arctic territory of Nunavut, Canada. Clinical Oncology, v. 32, n. I, p. 60-67, 2020.

CORREA-GALENDI, J. S. et al. Economic Modelling of Screen-and-Treat Strategies for Brazilian Women at Risk of Hereditary Breast and Ovarian Cancer. Applied Health Economics and Health Policy, p. I-I3, 2020.

DALCUCHE, Marise Gnatta; MENDES, Eugênio Vilaça. A integração em Rede da Atenção Primária (APS) com a Atenção Ambulatorial Especializada (AAE) no estado do Paraná. Espaço para Saúde, v. I8, n. I, p. 3I-37, 2017.

ELIAS, Juliana Costa et al. Levantamento dos custos do diagnóstico e tratamento oncológico no paciente idoso. Nursing (São Paulo), v. 23, n. 270, p. 4808-48I 5, 2020.

FIDLER, M. M. et al. Cancer incidence and mortality among young adults aged 20-39 years worldwide in 2012: a population-based study. The Lancet Oncology, v. I8, n. I2, p. I579-I589, 2017. 
FIGUEIREDO, F. W. S. et al. Socioeconomic changes in Brazil impacted breast cancer indexes at the beginning of the 2Ist century?. Journal of cancer policy, v. I6, p. 39-42, 2018.

FIGUEIREDO, Juliana Oliveira et al. Gastos público e privado com saúde no Brasil e países selecionados. Saúde em Debate, v. 42, p. 37-47, 2018.

HASSETT, M. J. et al. Spending for Advanced Cancer Diagnoses: Comparing Recurrent Versus De Novo Stage IV Disease. Journal of oncology practice, v. I5, n. 7, p. e616-e627, 2019.

INSTITUTO BRASILEIRO DE GEOGRAFIA E ESTATÍSTICA (IBGE). Síntese de indicadores sociais: uma análise das condições de vida da população brasileira - 20I7. Rio de Janeiro: IBGE, 2017

INSTITUTO NACIONAL DO CÂNCER (INCA). Atlas online de mortalidade. Disponível em: $<$ https://mortalidade.inca.gov.br/MortalidadeWeb/pages/Modelo04/consultar.xhtml\#panelResulta do $>$. Acesso em: 12 de dezembro de 2020.

KALIKS, Rafael Aliosha et al. Diferenças no tratamento sistêmico do câncer no Brasil: meu SUS é diferente do teu SUS. Braz J Oncol, v. I3, n. 44, p. I-I2, 2017.

MARQUES, Cristiane da Câmara et al. Casos de tuberculose coinfectados por HIV em um estado do nordeste brasileiro. Enfermería Actual de Costa Rica, n. 36, p. 62-76, 2019.

MENDES, José Dínio Vaz; BITTAR, Olímpio J. Nogueira V. Perspectivas e desafios da gestão pública no SUS. Revista da Faculdade de Ciências Médicas de Sorocaba, v. I6, n. I, p. 35-39, 20 I4.

MENDEZ, L. C. et al. Cancer deaths due to lack of universal access to radiotherapy in the Brazilian Public Health System. Clinical Oncology, v. 30, n. I, p. e29-e36, 2018.

MINISTÉRIO DA SAÚDE (BR). Sistema de Legislação da Saúde. SLEGIS. (2020) Disponível em: $<$ http://saudelegis.saude.gov.br/saudelegis/secure/norma/listPublic.xhtml>. Acesso em: 02 de janeiro de $202 \mathrm{I}$.

OBSERVATÓRIO DE ONCOLOGIA. 30 anos de SUS Saúde no Brasil e a evolução do tratamento do câncer. (2018). Disponível em:

$<$ http://observatoriodeoncologia.com.br/outros_estudos/IQVIA.pdf>. Acesso em: 06 de abril de 202I.

OCKÉ-REIS, C. O.; FERNANDES, A. M. P. Descrição do gasto tributário em saúde: 2003 a 2015. 2018.

OCKÉ-REIS, C. O.; MARMOR, T. R. The Brazilian national health system: an unfulfilled promise?. The International journal of health planning and management, v. 25, n. 4, p. 3I8-329, 2010.

PAIM, J. et al. The Brazilian health system: history, advances, and challenges. The Lancet, v. 377, n. 9779, p. I778-I797, 20II.

PFLUEGER, Dane. Accounting for quality: on the relationship between accounting and quality improvement in healthcare. BMC Health Services Research, v. I5, n. I, p. I-I3, 2015. 
PIOLA, Sérgio Francisco et al. Estruturas de financiamento e gasto do sistema público de saúde. Rio de Janeiro: Fundação Oswaldo Cruz, 2013.

PIOLA, Sergio Francisco; BENEVIDES, Rodrigo Pucci de Sá; VIEIRA, Fabiola Sulpino. Consolidação do gasto com ações e serviços públicos de saúde: trajetória e percalços no período de 2003 a 2017. 2018.

SALAS-VEGA, S.; MOSSIALOS, E. Cancer drugs provide positive value in nine countries, but the United States lags in health gains per dollar spent. Health Affairs, v. 35, n. 5, p. 8I3-823, 2016.

SANTOS, C. L. et al. Estimation of the Costs of Invasive Cervical Cancer Treatment in Brazil: A Micro-Costing Study. Revista Brasileira de Ginecologia e Obstetrícia, v. 4I, n. 6, p. 387-393, 2019.

SANTOS, Lenir. Região de saúde e suas redes de atenção: modelo organizativo-sistêmico do SUS. Ciência \& Saúde Coletiva, v. 22, p. I28I-I289, 2017.

SANTOS NETO, João Alves dos et al. Análise do financiamento e gasto do Sistema Único de Saúde dos municípios da região de saúde Rota dos Bandeirantes do estado de São Paulo, Brasil. Ciência \& Saúde Coletiva, v. 22, p. I269-I280, 2017.

SILVA, Dina Carla Vasconcelos Sena; VACOVSKI, Eduardo. A transparência na administração pública como instrumento facilitador para o controle social. Gestão Pública, v. 7, n. 4, 2018.

SILVA, Lívia Christina Almeida et al. Contribuições da atenção farmacêutica á pacientes em tratamento oncológico. Revista de Investigação Biomédica, v. 9, n. 2, p. 210-217, 2018.

SILVA, Mariana Cristina Lobato dos Santos Ribeiro; SILVA, Lucía; BOUSSO, Regina Szylit. A abordagem à família na Estratégia Saúde da Família: uma revisão integrativa da literatura. Revista da Escola de Enfermagem da USP, v. 45, n. 5, p. I250-I255, 201 I.

SIQUEIRA, A. S. E. et al. Economic impact analysis of cancer in the health system of Brazil: model based in public database. Health Science Journal, v. I I, n. 4, p. I, 2017.

SOUZA, Diego de Oliveira. Financeirização, fundo público e os limites à universalidade da saúde. Saúde em debate, v. 43, n. spe5, p. 7I-8I, 2019.

TEIXEIRA, Luiz Antonio et al. Políticas públicas de controle de câncer no Brasil: elementos de uma trajetória. 2012.

VIEIRA, Fabiola Sulpino; PIOLA, Sergio Francisco; BENEVIDES, Rodrigo Pucci de Sá. Vinculação orçamentária do gasto em saúde no Brasil: Resultados e argumentos a seu favor. 2019.

ZAREMBA, G. et al. Out-of-pocket costs for cancer patients treated at the Brazilian public health system (SUS) and for their caregivers: A pilot study. Clinical Oncology Letters, v. 2, n. I, p. 23-30, 2019. 